\title{
Does Ovulation Induction Increase the Risk of Aneuploid Conception? Comparison of First Trimester Miscarriages after FSH Stimulated Cycles and Naturally Conceived Cycles
}

\author{
Celtemen Pinar Telli ${ }^{1 *}$, Erdem Mehmet ${ }^{1}$, Bozkurt Nuray $^{1}$, Meral Yirmibes $^{2}$, Oktem Mesut $^{1}$, \\ Erdem Ahmet ${ }^{1}$, Karabacak Onur' ${ }^{1}$, Celtemen Mustafa Baran ${ }^{3}$, Kayhan Gülsüm ${ }^{2}$
}

\begin{abstract}
Objective: The aim of this study was to determine the rate of chromosomal abnormalities in the products of first trimester miscarriages in patients who were under FSH ovarian stimulation and to compare the results with the products of miscarriages of naturally conceived pregnancies.

Materials and Methods: This retrospective cohort study was conducted over a nine-year period. Included were 909 patients who conceived naturally and 76 infertility patients who were under FSH stimulation with first trimester miscarriages karyotyped. The rate of aneuploidy was compared between the two groups. The main outcome measure was the incidence of aneuploidy.

Results: In 76 FSH-induced pregnancies, 12 (15.7\%) had an abnormal karyotype. The rate of aneuploidy rate in miscarriages in the natural conception group was $22.3 \%$. There was no statistically significant difference in age (32.7 \pm 4.2 vs. $33.5 \pm 5.0)$, cycle day 3 FSH $(7.9 \pm 2.3 \mathrm{mIU} / \mathrm{ml}$ vs. $7.1 \pm 2.6 \mathrm{mIU} / \mathrm{ml})$ or exogenous FSH doses $(2419.7 \pm 643.7 \mathrm{IU}$ vs. $2616.5 \pm 947.5 \mathrm{IU})$ between the infertile patients with or without abnormal karyotypes.

Conclusion: Exposure to exogenous FSH was not associated with increased aneuploidy rates in our infertile population.

Keywords: Aneuploidy, IVF, Miscarriage, Menarche, Ovulation Induction
\end{abstract}

\section{Introduction}

Up to $30 \%$ of human embryos fail to survive (1). Embryonic aneuploidy is the main cause of spontaneous abortion in approximately $65 \%$ of cases $(2,3)$. High rates of numerical chromosome abnormalities or aneuploidies may also be associated in conceptions after assisted reproductive therapy (ART) $(4,5)$. Although the rate of occurrence increases with maternal age, semen quality and the morphological quality of the embryos could also be associated with aneuploidy (6).

The exogenous follicle stimulating hormone (FSH) is often administered to induce multiple follicle growth in ART. Evidence has suggested that elevated levels of endogen $(\geq 20 \mathrm{ng} / \mathrm{ml}$ ) and exogenous gonadotropins might cause chromosomal abnormalities $(7,8)$. It was hypothesized that "hormonal imbalance causes an increased risk of non-disjunction by affecting oocyte ripeness, the rate of meiosis and spindle integrity" $(8,9)$. Another explanation could be the recruitment of poor quality oocytes when the process of the natural selection of a dominant follicle is overridden by ovarian hyperstimulation. Indeed, the cause of miscarriages in the first trimester of the pregnancy is closely related to aneuploidy.
Animal and human studies demonstrated in that sudden elevation of FSH damages chromosomes and affects embryo quality (10-14), but no studies have shown a direct link between gonadotropins and the genesis of aneuploidy. To test the hypothesis that exogenous gonadotropins might be related to aneuploidy, we aimed to determine the rate of chromosomal abnormalities in the products of first trimester miscarriages in patients who were pregnant after FSH ovarian stimulation and those of miscarriages of naturally conceived pregnancies.

\section{Materials and Methods}

This was a retrospective cohort study conducted over nine years. The subjects included 985 patients whose abortion materials were karyotyped. Nine hundred and nine of these materials were obtained from naturally conceived pregnancies and 76 were obtained from infertile patients who were pregnant under FSH hyperstimulation in invitro fertilization (IVF).

Miscarriage was defined as loss of clinical pregnancy after identification of gestational sac on transvaginal ultrasound. Chorionic villi were carefully dissected from the remaining tissue during the termination procedure, 
which was performed by either suction or dilatation and curettage. Patients with recurrent miscarriages (3), frozen embryo transfer, and patients who conceived after testicular sperm extraction were excluded. Some minced chorionic villi material was used to initiate two or three separate cell cultures. Colchicine was added two hours before the cytologic preparation. Harvesting and Giemsa banding of metaphase chromosomes were performed. Five metaphase spreads were analyzed; fifteen metaphase spreads were counted for each one of the specimens. The rate of aneuploidy was compared between patients who conceived after FSH treatment with patients who conceived naturally. The main outcome measure was the rate of chromosomal abnormalities. Variables that may affect embryonic karyotype, such as age, basal FSH, and total FSH dose were also compared between cases with normal and abnormal karyotypes.

Three ovarian hyperstimulation protocols were used.

GnRH agonist long protocol: One mg daily leuprolide was given at least for 14 days from subcutaneous route. Gonadotropin treatment was begun if the serum estradiol level was lower than $50 \mathrm{pg} / \mathrm{ml}$ at the mid-luteal phase (on the 21st day) of the cyclus prior to gonadotropin was begun and menstrual bleeding occurred. Leuprolide acetate (Lucrin flacon ${ }^{\circledR} 1 \mathrm{mg}$ Abbott, France) treatment was decreased to dose of $0.5 \mathrm{mg} /$ day and continued with the same dose during the gonadotropin (FSH) treatment. Microdose flare-up protocol: Oral contraceptive treatment consisting ethinyl estradiol $0.03 \mathrm{mg}+$ levonorgestrel $0.150 \mathrm{mg}$ (Microgynon tablet ${ }^{\circledR}$, Schering AG Berlin) daily was given between the days 1 and 21 of the prior cycle of gonadotropin treatment. Leuprolide acetate (Lucrin flacon ${ }^{\circledR} 1 \mathrm{mg}$ Abbott, France) 40 microgram bid from subcutaneous route was begun after 2 days from oral contraceptive drug was stopped. Gonadotropin stimulation was begun at a suitable dose after the day leuprolide acetate was begun. Pituitary suppression was continued with the same dose during gonadotropin stimulation until the day of hCG.

GnRH Antagonist protocol: Gonadotropin stimulation was begun at the 3rd day of the cycle with the appropriate dose if the cystic lesion was not found with ultrasonography without pituitary suppression treatment. Cetrorelix (Cetrotide flakon ${ }^{\circledR}$, Merck Serono, Istanbul, Turkey) $0.25 \mathrm{mg} /$ day from subcutaneous route was begun when the dominant follicle reached to $14 \mathrm{~mm}$ diameter and given with gonadotropins until the day of hCG.

Recombinant gonadotropin FSH (GONAL- $\mathrm{f}^{\circledR}$, Merck Serono) dose was ordered due to ovarian response in all protocols.

Chi square and Mann-Whitney $U$ tests were used for the statistical analysis. $\mathrm{P}$ values of $<0.05$ were regarded as statistically significant.

\section{Results}

Among 985 specimens, 215 (21.8\%) were abnormal. Twelve of 76 FSH-induced pregnancies had an abnormal karyotype consisting of double and triple aneuploidies; 3 with $45, \mathrm{X}$ and $47, \mathrm{XY},+15$; others were $47, \mathrm{XX},+21$, $48, \mathrm{XX}+15,+20,47, \mathrm{XY},+3,47, \mathrm{XX},+2,49, \mathrm{XY},+8,+20,+21$, $47, \mathrm{XX},+16, \quad 47, \mathrm{XX},+18$. The results showed no statistically significant differences in aneuploidy rates between FSH induced group and natural conceived group ( $\mathrm{p}>0.05$; Table 1 ).

The mean age was older in the FSH-induced group, compared to the natural conception group $(\mathrm{p}<0.05)$. However, the mean age was similar in patients with abnormal $(31.8 \pm 5.8)$ and normal karyotypes $(31.0 \pm 5.4)$ in the whole study population $(\mathrm{p}>0.05)$. When patients were categorized according to age, in the natural conception group, the incidence of aneuploidy was significantly lower in patients $<35$ years, compared to patients $>35(19.7 \%$ vs. $28.4 \%, \mathrm{p}=0.008)$. In the FSH-induced pregnancy group, the difference between aneuploidy rates were not significantly different in patients below (16.3\%) or above (14.8\%) 35 years of age.

The characteristics of patients under FSH stimulation are shown in Table 2 . There were no statistically significant differences in age, cycle-day 3 FSH, and exogenous FSH doses between the infertile patients with or without abnormal karyotypes $(\mathrm{p}>0.05)$.

Table 1. Comparison of natural conception and FSH-induced conception.

\begin{tabular}{lccc}
\hline & Natural Conception & FSH-induced conception & p-value \\
\hline Maternal age (y) & $31.0 \pm 5.5$ & $33.4 \pm 4.8$ & $\mathrm{p}<0.05$ \\
Abnormal karyotype (\%) & 22.3 & 15.7 & $\mathrm{p}>0.05$ \\
\hline
\end{tabular}

Table 2. Characteristics of patients under FSH stimulation, euploid versus aneuploid fetal karyotypes.

\begin{tabular}{lccc}
\hline & $\begin{array}{c}\text { Aneuploid } \\
\mathbf{N}=\mathbf{1 2}\end{array}$ & $\begin{array}{c}\text { Euploid } \\
\mathbf{N}=\mathbf{6 4}\end{array}$ & p-value \\
\hline Maternal age (y) & $32,7 \pm 4,2$ & $33,5 \pm 5,0$ & $>0.05$ \\
Cycle Day 3 E2 (pg/mL) & $46,1 \pm 16,6$ & $41,5 \pm 17,3$ & $>0.05$ \\
Cycle Day 3 FSH (IU/L) & $7,9 \pm 2,3$ & $7,1 \pm 2,6$ & $>0.05$ \\
Total FSH dose (IU) & $2419,7 \pm 643,7$ & $2616,5 \pm 947,5$ & $>0.05$ \\
\hline
\end{tabular}




\section{Discussion}

The results of this study showed that the incidence of aneuploidy did not differ in the conceptions of patients under FSH stimulation for the treatment of infertility. Previous studies have shown controversial results regarding this issue. High doses of exogenous FSH stimulation were related to decreases in embryo quality and increases in aneuploidy rates in some studies (10-12, 15). High concentrations of FSH in in-vitro maturation (IVM) medium were found to increase the first meiotic division error, resulting in an increased number of aneuploid oocytes $(7,14)$. Mild ovarian stimulation in IVF was found to stimulate the growth of the most mature follicles, allowing a degree of selection of oocytes in decreased proportion to aneuploidy $(1,12,14)$.

Exposure to exogenous gonadotropins could possibly be related to increased chromosomal abnormalities in conceptions after treatment of IVF. The exact mechanism by which FSH induces aneuploidy remains unclear, but several explanations have been suggested, as follows: Exaggerated gonadotropin exposure may cause damage in meiotic progression caused by uncontrolled follicular growth and oocyte susceptibility to the environment during the first meiosis. FSH may affect microtubules, which may have a role in meiosis and meiotic spindle formation (8). Theoretically, it could also be assumed that only one best-quality oocyte is selected during the natural cycle in humans. FSH may rescue abnormal oocytes and facilitate the meiotic resumption of these oocytes, which otherwise may be destined for atresia (14).

Recent data demonstrated no difference between exogenous FSH exposure and embryonic aneuploidy, which was compatible with our results $(13,14,16-19)$. In our study, total FSH dose and cycle-day 3 FSH levels were similar in patients with abnormal and normal karyotypes. We found that exogenous gonadotropin administration was not associated with the increased risk of aneuploidy in patients having gonadotropin stimulation for infertility. It is well known that advanced maternal age is related to an increased rate of chromosomal abnormalities in embryos. The increased rate of aneuploidy might be the result of increased endogenous gonadotropins in advanced maternal age (8). Some investigators proposed that remaining oocytes may have higher probability for chromosomal abnormalities because of the long incubation period from meiosis I to meiosis II (8). However, our study population represents a relatively young group of infertile patients and control patients (mean age of the population was $31.2 \pm 5.5$ ).

Increase the risk of aneuploidy in women treated for infertility.

\section{Conclusion}

In conclusion, in this study the incidence of aneuploidy in the cytogenetic analysis of conception products was not higher in pregnancies conceived with FSH stimulation, compared with spontaneous conceptions. The findings suggest that exogenous FSH used for stimulation does not increase the risk of aneuploidy in women treated for infertility.

\section{Ethical issues}

All patients signed an informed consent for genetic karyotyping. Approval of the local ethics committee from "Gazi University Clinical Research Ethics Committee" was taken before the study was begun.

\section{Conflict of interests}

We declare that we have no conflict of interests.

\section{Acknowledgments}

We have no acknowledgements to disclose.

\section{References}

1. Baart EB, Martini E, Eijkemans MJ, Van Opstal D, Beckers NG, Verhoeff A, et al. Milder ovarian stimulation for in-vitro fertilization reduces aneuploidy in the human preimplantation embryo: a randomized controlled trial. Hum Reprod 2007; 22:980-8.

2. Nybo-Andersen AM, Wohlfahrt J, Christens P, Olsen J, Melbye M. Maternal age and fetal loss: population based register linkage study. BMJ 2000;320:1708-12.

3. Lathi RB, Mark SD, Westphal LM, Milki AA. Cytogenetic testing of anembryonic pregnancies compared to embryonic missed abortions. J Assist Reprod Genet 2007;24:521-4.

4. Lathi RB, Milki AA. Rate of aneuploidy in miscarriages following in vitro fertilization and intracytoplasmic sperm injection. Fertil Steril 2004;81:5.

5. Twisk M, Mastenbroek S, Hoek A, Heineman MJ, Veen F, Bossuyt PM, et al. No beneficial effect of preimplantation genetic screening in women of advanced maternal age with a high risk for embryonic aneuploidy. Human Reprod 2008;23:2813-7.

6. Fragouli E, Wells D. Aneuploidy in the Human Blastocyst. Cytogenet Genome Res 2011; 133:149-59.

7. Roberts R, Iatropoulou A, Ciantar D, Stark J, Becker DL, Franks S. Follicle-stimulating hormone affects metaphase I chromosome alignment and increases aneuploidy in Mouse oocytes matured in vitro. Biol Reprod 2005; 72:107-18.

8. Dursun P, Gultekin M, Yuce K, Ayhan A. What is the underlying cause of aneuploidy associated with increasing maternal age? Is it associated with elevated levels of gonadotropins? Med Hypoth 2006; 66:143-7.

9. Gaulden M. Maternal age effect: the enigma of Down syndrome and other trisomic conditions. Mutat Res 1992; 296:68-9.

10. Edgar DH, Whalley KM, Mills JA. Effects of highdose and multiple-dose gonadotropin stimulation on mouse oocyte quality as assessed by preimplantation development following in vitro fertlization. J In Vitro Fert Embryo Transf 1987; 4:273-6.

11. Sato F, Marrs RP. The effect of pregnant mare serum gonadotropin on mouse embryos fertilized in vivo or in vitro. J In Vitro Fert Embryo Transf 1986; 3:353-7.

12. Baart EB, Martini E, Eijikemans MJ, van Opstal D, Beckers NG, Verhoeff A. Milder ovarian stimulation for in-vitro fertilization reduces aneuploidy in the human preimplantation embryo: a randomized controlled trial. 
Hum Reprod 2007; 22:980-8.

13. Xu L, Wang Y, Zhou P, Cao YX, Huang TH, Chian RC. Cytogenetic analysis of in vivo and in vitro oocytes derived from naturally cycling and stimulated mice. Syst Biol Reprod Med 2008; 54:155-62.

14. Xu YW, Peng YT, Wang B, Zeng YH, Zhuang GL, Zhou CQ. High follicle-stimulating hormone increases aneuploidy in human oocytes matured in vitro. Fertil Steril 2011; 95:1

15. Elbling L, Colot M. Abnormal development and transport and increased sister-chromatid Exchange in preimplantation embryos following superovulation in mice. Mutat Res 1985;147:189-95.

16. Massie JA, Shahine LK, Milki AA, Westphal LM, Lathi RB. Ovarian stimulation and the risk of aneuploid conceptions.
Fertil Steril 2011; 95:3.

17. Verpoest W, Fauser BC, Papanikolaou E, Staessen C, Van Landuyt L, Donoso P, et al. Chromosomal aneuploidy in embryos conceived with unstimulated cycle IVF. Hum Reprod 2008; 23:2369-71.

18. Martinez MC, Mendez C, Ferro J, Nicolas M, Serra $\mathrm{V}$, Landeras J. Cytogenetic analysis of early nonviable pregnancies after assisted reproduction treatment. Fertil Steril 2010; 93:289-92.

19. Braga DP, Setti AS, Figueira Rde C, Iaconelli A Jr, Borges E Jr. Contributing factors for the incidence of aneuploidy in older patients undergoing intracytoplasmic sperm injection cycles. J Assist Reprod Genet 2012; 29:911-6.

Copyright $(2014$ The Author(s); This is an open-access article distributed under the terms of the Creative Commons Attribution License (http://creativecommons.org/licenses/by/4.0), which permits unrestricted use, distribution, and reproduction in any medium, provided the original work is properly cited. 